\title{
Generalized Dual-Mode Index Modulation Aided OFDM
}

\author{
Tianqi Mao, Student Member, IEEE, Qi Wang, Member, IEEE, and Zhaocheng Wang, Senior Member, IEEE
}

\begin{abstract}
Dual-mode index modulation aided orthogonal frequency division multiplexing (DM-OFDM) is recently proposed, where subcarriers are partitioned into OFDM subblocks, divided into two groups within each subblock, and modulated by two differentiable constellation alphabets. In DM-OFDM, additional bits can be transmitted through indices of subcarriers modulated by the same constellation alphabet. In this letter, generalized DM-OFDM (GDM-OFDM) is proposed, where the number of subcarriers modulated by the same constellation mode in each subblock is alterable. By applying such enhancement, the spectral efficiency can be improved at the cost of marginal performance loss. Moreover, since the BER performance of GDM-OFDM degrades at low signal-to-noise ratios (SNRs), an interleaving technique is employed to address this issue. At the receiver, a maximum-likelihood (ML) detector and a reduced-complexity log-likelihood ratio (LLR) detector are employed for demodulation. Simulation results demonstrate that the proposed GDMOFDM is capable of enhancing the spectral efficiency compared with DM-OFDM at the cost of negligible performance loss, and the interleaved GDM-OFDM (IGDM-OFDM) can harvest on performance gain over GDM-OFDM.
\end{abstract}

Index Terms-Orthogonal frequency division multiplexing (OFDM), index modulation, maximum likelihood detection, loglikelihood ratio based detection, interleaving technique.

\section{INTRODUCTION}

Orthogonal frequency division multiplexing (OFDM) has emerged as a promising technology due to its enormous merits, including the high spectral efficiency and the resistance to the multipath fading [1]. Besides, it provides an effective solution against inter-symbol interference (ISI) caused by a dispersive channel [2]. Therefore, OFDM has been widely applied in wireless standards, including digital video broadcasting (DVB), $802.11 \mathrm{a} / \mathrm{g} \mathrm{Wi-Fi,} \mathrm{and} \mathrm{802.16} \mathrm{WiMAX} \mathrm{[1],} \mathrm{[3],} \mathrm{[4].}$

Index modulation has been recently introduced to OFDM, where the information bits are transmitted using not only amplitude/phase modulation techniques, but also the indices of the activated subcarriers [5], [6], [7]. The index modulation technique is capable of increasing the power efficiency of the classical OFDM, since only part of the subcarriers are modulated, and additional information bits can be conveyed by indices of the modulated subcarriers [8]. In [6], Basar et al. proposes OFDM with index modulation (OFDM-IM), which achieves performance gain compared with classical OFDM. However, it suffers from loss of the spectral efficiency, since only a fraction of subcarriers are modulated for

T. Mao and Z. Wang are with Tsinghua National Laboratory for Information Science and Technology (TNList), Department of Electronic Engineering, Tsinghua University, Beijing 100084, China (e-mail: mtq15@mails.tsinghua.edu.cn, zcwang@tsinghua.edu.cn).

Q. Wang is with School of Electronics and Computer Science, University of Southampton, Southampton SO17 1BJ, U.K. (e-mail: qw1e16@soton.ac.uk).

This work was supported in part by National Key Basic Research Program of China (Grant No. 2013CB329200), in part by Shenzhen Subject Arrangements (Grant No. JCYJ20160331184124954), in part by Shenzhen Peacock Plan (Grant No. 1108170036003286), in part by Shenzhen Fundamental Research Project (Grant No. JCYJ20150401112337177), and in part by EPSRC funded projects (Grant Nos. EP/N004558/1 and EP/N023862/1). data transmission. In [9] and [10], OFDM-IM is combined with MIMO techniques to achieve enhanced performance over classical MIMO systems. OFDM-IM is also applied to visible light communications [11] and underwater acoustic communications [12], which attains better BER performance than conventional OFDM schemes. Besides, the interleaving technique is combined with OFDM-IM in [13], [14] to further improve the performance of OFDM-IM. Furthermore, Fan et al. proposes generalized schemes of OFDM-IM in [15], where the number of activated subcarriers in each OFDM subblock is variable. Through such enhancement, the spectral efficiency can be significantly enhanced compared with OFDM-IM. Recently, dual-mode index modulation aided OFDM (DMOFDM) [16] has been proposed, where all the subcarriers are modulated by two distinguishable constellation alphabets. It is validated that DM-OFDM is capable of enhancing the BER performance of OFDM-IM at the same spectral efficiency.

In this letter, the generalized DM-OFDM (GDM-OFDM) is proposed, where the number of subcarriers modulated by the same constellation alphabet is alterable and determined by the information bits of each OFDM subblock. Since there are more possible realizations of the OFDM subblock in GDMOFDM than classical DM-OFDM, the total transmitted bits per subblock are increased, leading to the enhanced spectral efficiency. Moreover, an interleaving scheme is proposed to eliminate the correlation of the channel fading, attaining additional diversity gain in Rayleigh fading channels. At the receiver, a maximum likelihood (ML) detector and a reducedcomplexity log-likelihood ratio (LLR) detector are employed to demodulate the signals. Simulation results demonstrate that GDM-OFDM is capable of enhancing the spectral efficiency over classical DM-OFDM at the cost of negligible performance loss. Besides, the interleaved GDM-OFDM (IGDMOFDM) presents its superiority over DM-OFDM in both the BER performance and the spectral efficiency.

\section{DM-OFDM SySTEM MODEL}

At the transmitter, $N$ subcarriers are partitioned into $g$ subblocks of length $l=N / g$ [16]. The $\gamma$ th subblock can be formulated as $\mathbf{X}^{(\gamma)}=$ $[X((\gamma-1) l+1), X((\gamma-1) l+2), \cdots, X(\gamma l)]^{\mathrm{T}}, 1 \leq \gamma \leq$ $g$. For each OFDM subblock, two constellation sets $\mathcal{M}_{A}$ and $\mathcal{M}_{B}$ are employed for modulation, where $\mathcal{M}_{A} \wedge \mathcal{M}_{B}=\varnothing$. Subcarriers in each OFDM subblock are divided into two groups $\mathrm{A}$ and $\mathrm{B}$, where subcarriers in group $\mathrm{A}$ and $\mathrm{B}$ are modulated by $\mathcal{M}_{A}$ and $\mathcal{M}_{B}$ respectively. The two sets containing subcarrier indices of two groups are defined as $\mathcal{I}_{A}$ and $\mathcal{I}_{B}$, and $\mathcal{I}_{A}$ is referred to as the index pattern of the OFDM subblock, since $\mathcal{I}_{B}$ is determined as long as $\mathcal{I}_{\mathcal{A}}$ is known. It is indicated that additional information bits can be conveyed implicitly by the index pattern of each OFDM subblock. Hence, if $k$ subcarriers in each OFDM subblock 
are modulated by $\mathcal{M}_{A}$, and the sizes of $\mathcal{M}_{A}$ and $\mathcal{M}_{B}$ equal $N_{A}$ and $N_{B}$, the total transmitted bits per subblock can be formulated as

$$
n_{\mathrm{DM}}=\left\lfloor\log _{2}\left(\begin{array}{l}
l \\
k
\end{array}\right)\right\rfloor+k \log _{2}\left(N_{A}\right)+(l-k) \log _{2}\left(N_{B}\right) .
$$

The index modulation operations can be clearly illustrated by Table I, where $l=4$ and $k=2$. With the aid of an index selector, the index pattern $\mathcal{I}_{A}$ of each OFDM subblock can be determined by the $n_{1}=\log _{2}\left(\begin{array}{l}l \\ k\end{array}\right)=2$ index bits.

After the constellation mapping, an $N$-point IFFT operation is performed on the $g$ subblocks, generating time-domain (TD) signals $\mathbf{x}=[x(1), x(2), \cdots, x(N)]$. In this letter, the frequency-selective Rayleigh fading channel [6] is considered, where the length of the channel impulse response (CIR) $L_{h}$ is no larger than the CP length $L_{c p}$. The TD CIR coefficients can be represented by $\mathbf{h}=\left[h(1), h(2), \cdots, h\left(L_{h}\right)\right]$, where $h(i)$ denotes the circularly symmetric complex Gaussian random variables, with the distribution of $\mathcal{C N}\left(0,1 / L_{c p}\right)$.

\section{Proposed Generalized DM-OFDM}

Since the size of $\mathcal{I}_{A}$ is fixed in classical DM-OFDM, the possible realizations of OFDM subblocks can be increased by implementing various sizes of $\mathcal{I}_{A}$ in different subblocks, harvesting on higher spectral efficiency. Therefore, GDMOFDM is proposed to enhance the spectral efficiency of DMOFDM, where the number of subcarriers modulated by $\mathcal{M}_{A}$ is no longer fixed, and the sizes of $\mathcal{I}_{A}$ and $\mathcal{I}_{B}$ are determined by the information bits. For each OFDM subblock, $\mathcal{K}$ is defined as the set containing possible sizes of $\mathcal{I}_{A}$. It is represented by $\mathcal{K}=\left\{k_{1}, k_{2}, \cdots, k_{T}\right\}$, where $T$ is the size of $\mathcal{K}$. Unlike classical DM-OFDM, the index bits fed into the index selector is firstly used to determine the size of $\mathcal{I}_{A}$, denoted as $k_{i}$ $(1 \leq i \leq T)$, then $k_{i}$ subcarriers are selected to be modulated by $\mathcal{M}_{A}$, whilst the other $\left(l-k_{i}\right)$ subcarriers are modulated by $\mathcal{M}_{B}$. Therefore, the number of possible OFDM subblock realizations is derived as $n_{r}=\sum_{i=1}^{T}\left(\begin{array}{c}l \\ k_{i}\end{array}\right) N_{A}^{k_{i}} N_{B}^{l-k_{i}}$. And the total information bits transmitted by each OFDM subblock is calculated as

$$
n_{\mathrm{GDM}}=\left\lfloor\log _{2}\left(\sum_{i=1}^{T}\left(\begin{array}{c}
l \\
k_{i}
\end{array}\right) N_{A}^{k_{i}} N_{B}^{l-k_{i}}\right)\right\rfloor .
$$

Whilst for classical DM-OFDM, subcarriers modulated by $\mathcal{M}_{A}$ in each subblock is restricted to a constant $k_{A}$. Hence, the total information bits conveyed by each subblock can be represented as $n_{\mathrm{DM}}^{\prime}=\left\lfloor\log _{2}\left(\left(\begin{array}{c}l \\ k_{A}\end{array}\right) N_{A}^{k_{A}} N_{B}^{l-k_{A}}\right)\right\rfloor$.

Since GDM-OFDM has much more possible realizations of OFDM subblocks, its spectral efficiency can be improved compared with classical DM-OFDM. For example, we assume that the length of subblocks is 4, and two differentiable quadrature phase shift keying (QPSK) are employed for modulation. For GDM-OFDM, given that $\mathcal{K}=\{1,3\}$ and $\{0,1,2,3,4\}$, the number of information bits for each subblock is calculated to be 11 bits and 12 bits, respectively. Correspondingly, for the DM-OFDM counterpart of Table I, each subblock can only transmit 10 bits. From (2), it is indicated that enlarging the size of $\mathcal{K}$ is capable of enhancing the spectral efficiency of GDMOFDM, and the maximum spectral efficiency can be achieved
TABLE I A look-up table of index modulation for $l=4$ and $k=2$.

\begin{tabular}{|c|c|c|}
\hline Index bits & Indices & Subblocks \\
\hline$[0,0]$ & {$[1,2]$} & {$\left[S_{A}, S_{A}^{\prime}, S_{B}, S_{B}^{\prime}\right]$} \\
\hline$[0,1]$ & {$[2,3]$} & {$\left[S_{B}, S_{A}, S_{A}^{\prime}, S_{B}^{\prime}\right]$} \\
\hline$[1,0]$ & {$[3,4]$} & {$\left[S_{B}, S_{B}^{\prime}, S_{A}, S_{A}^{\prime}\right]$} \\
\hline$[1,1]$ & {$[1,4]$} & {$\left[S_{A}, S_{B}, S_{B}^{\prime}, S_{A}^{\prime}\right]$} \\
\hline
\end{tabular}

when $\mathcal{K}=\{0,1,2, \cdots, l\}$. However, GDM-OFDM may suffer from performance loss and large computational complexity due to the increased number of subblock realizations. Therefore, a tradeoff is required between the spectral efficiency and the BER performance as well as the computational complexity.

At the receiver, the frequency-domain (FD) signals $\mathbf{X}_{\mathbf{r}}=$ $\left[X_{r}(1), X_{r}(2), \cdots, X_{r}(N)\right]$ is demodulated by a ML detector. Define $\mathcal{X}$ as the set containing all the realizations of the OFDM subblock. The transmitted symbols and its corresponding index pattern for the $\gamma$ th subblock can be calculated by minimizing the following metric as

$\left[\mathcal{I}_{A}^{(\gamma)}, \hat{\mathbf{X}}^{(\gamma)}\right]=\arg \min _{\hat{\mathbf{X}}(\gamma) \in \mathcal{X}} \sum_{i=1}^{l}\left\|X_{r}^{(\gamma)}(i)-H^{(\gamma)}(i) \hat{X}^{(\gamma)}(i)\right\|^{2}$,

where $X_{r}^{(\gamma)}(i)=X_{r}(\gamma l+i), H^{(\gamma)}(i)=H(\gamma l+i)$, and $\mathbf{H}=[H(1), H(2), \cdots, H(N)]$ representing the FD channel coefficients. After the detection, the information bits transmitted by the constellations and the index pattern can be readily demodulated using a look-up table that maps the realizations of OFDM subblocks to the information bits. From (3), the computational complexity in terms of multiplications is on the order of $O\left(\sum_{i=1}^{T}\left(\begin{array}{c}l \\ k_{i}\end{array}\right) N_{A}^{k_{i}} N_{B}^{l-k_{i}}\right)$. Due to its exponentially increasing complexity, the ML detector tends to be inefficient as the coefficients $N_{A}, N_{B}, T$, and $l$ become larger.

To address the complexity issue, a reduced-complexity log-likelihood ratio (LLR) based detector is proposed. Each subcarrier is either modulated by $\mathcal{M}_{A}$ or by $\mathcal{M}_{B}$. Hence, in order to obtain the transmitted symbol of the $\alpha$ th subcarrier for $\alpha=1,2, \cdots, N$, the logarithm of the ratio between the posteriori probabilities of whether the subcarrier is modulated by $\mathcal{M}_{A}$ or by $\mathcal{M}_{B}$ is calculated as

$$
\eta(\alpha)=\ln \left(\frac{\sum_{j=1}^{N_{A}} P\left(X(\alpha)=S_{A}^{(j)} \mid X_{r}(\alpha)\right)}{\sum_{q=1}^{N_{B}} P\left(X(\alpha)=S_{B}^{(q)} \mid X_{r}(\alpha)\right)}\right),
$$

where $S_{A}^{(j)}$ denotes the $j$ th element of $\mathcal{M}_{A}$ and $S_{B}^{(q)}$ is the $q$ th constellation of $\mathcal{M}_{B}$. Since the size of $\mathcal{I}_{A}$ is $k_{i}$, (4) can be further simplified using the Bayes' formula as [16]

$$
\begin{aligned}
& \eta(\alpha)= \\
& \ln \left(\frac{N_{B} k_{i}}{N_{A}\left(l-k_{i}\right)}\right)+\ln \left(\sum_{j=1}^{N_{A}} \exp \left(-\frac{\left|X_{r}(\alpha)-H(\alpha) S_{A}^{(j)}\right|^{2}}{N_{0}}\right)\right) \\
& -\ln \left(\sum_{q=1}^{N_{B}} \exp \left(-\frac{\left|X_{r}(\alpha)-H(\alpha) S_{B}^{(q)}\right|^{2}}{N_{0}}\right)\right)
\end{aligned}
$$


For each $k_{i}$ where $i=1,2, \cdots, T$, the LLR values of subcarriers are calculated. It is indicated that one subcarrier is more probably modulated by $\mathcal{M}_{A}$ if the corresponding LLR value is positive, and vice versa. Therefore, the index pattern for $k_{i}$, denoted as $\mathcal{I}_{A k_{i}}$, can be determined by the positive and negative signs of the LLR values in each subblock, and the corresponding symbols $\hat{\mathbf{X}}_{k_{i}}=\left[\hat{X}_{k_{i}}(1), \hat{X}_{k_{i}}(2), \cdots, \hat{X}_{k_{i}}(l)\right]$ are obtained using hard decision.

Remark: Due to the effect of noise, there exists an illegal index pattern after LLR calculation for a particular OFDM subblock, where the number of subcarriers with positive LLR values is not included in $\mathcal{K}$. To address the issue, assuming that the number of subcarriers modulated by the mapper $\mathrm{A}$ in the OFDM subblock is $k_{i}(1 \leq i \leq T)$, then the subcarriers corresponding to the $k_{i}$ largest LLR values in each OFDM subblock are considered to be modulated by the mapper A, instead of judging the signs of LLR values.

After that, the optimal constellation symbols and the corresponding index pattern for the $\gamma$ th OFDM subblock among all the possible $\mathcal{I}_{A k_{i}}$ and the $\hat{\mathbf{X}}_{k_{i}}$ for $i=1,2, \cdots, T$ can be determined by minimizing the following ML metric as

$$
\left[\mathcal{I}_{A \hat{k}}, \hat{\mathbf{X}}_{\hat{k}}\right]=\arg \min _{\hat{k} \in \mathcal{K}} \sum_{q=1}^{l}\left\|X_{r}^{(\gamma)}(q)-H^{(\gamma)}(q) \hat{X}_{\hat{k}}(q)\right\|^{2}
$$

After the detection, information bits can be easily demodulated using a look-up table, which is similar to the operations of the proposed ML detector. Although the reduced-complexity LLR detector is near-optimal, the computational complexity in terms of complex multiplication is on the order of $l T\left(N_{A}+N_{B}\right)$ per subblock, which is much lower than the ML detection. Hence, the LLR detector is preferred for practical GDM-OFDM systems with large $N_{A}, N_{B}, T$, and $l$.

When the Rayleigh fading channel is considered, GDMOFDM may suffer from performance loss due to the highlycorrelated channel fading [13]. To reduce the correlation of FD channel coefficients, an interleaving technique is applied to GDM-OFDM, where the $g \times l$ matrix consist of $g$ subblocks is passed through an interleaver $\prod_{L \times g}$ before being fed into the IFFT module in a row-by-row manner. At the receiver, after the FFT operation, the FD signals and the channel coefficients $\mathbf{H}$ are firstly deinterleaved and then processed by the detector. The BER performance of IGDM-OFDM is believed to be significantly improved compared with GDM-OFDM, since the signals in each OFDM subblock can be transmitted through uncorrelated channels to attain additional diversity gain.

\section{Simulation Results And Discussion}

The performance of GDM-OFDM is compared with DMOFDM under both the frequency-selective fading channel and the additive white Gaussian noise (AWGN) channel, where the ML detector and the LLR detector are employed. Moreover, the performance of the interleaving technique is also investigated. In the simulations, the IFFT size $N$ equals 128 . For the size of OFDM subblocks, since the computational complexity of the ML detector for both the proposed GDM-OFDM and DM-OFDM increases exponentially with the subblock size, it is set as a relatively small value of 4 for practical use. Besides, the lengths of the CIR coefficients and the CP are 10 and 16.

The performance comparison between GDM-OFDM and DM-OFDM using the ML and LLR detectors under the Rayleigh fading channel and the AWGN channel is depicted in Fig. 1 , where $\mathcal{M}_{A}=\{-1-\mathrm{j}, 1-\mathrm{j}, 1+\mathrm{j},-1+\mathrm{j}\}$ and $\mathcal{M}_{B}=$ $\{1+\sqrt{3},(1+\sqrt{3}) \mathrm{j},-1-\sqrt{3},-(1+\sqrt{3}) \mathrm{j}\}$. The proposed GDM-OFDM with $\mathcal{K}=\{1,3\}$ harvests on a $0.22 \mathrm{bits} / \mathrm{s} / \mathrm{Hz}$ spectral efficiency gain over conventional DM-OFDM, and even a $0.8 \mathrm{~dB}$ SNR gain is attained under the AWGN channel, because higher spectral efficiency can reduce $E_{b}$, yielding smaller needed $E_{b} / N_{0}$. Besides, under the Rayleigh fading channel, GDM-OFDM with $\mathcal{K}=\{0,1,2,3,4\}$ achieves even higher spectral efficiency of $2.67 \mathrm{bits} / \mathrm{s} / \mathrm{Hz}$ at the cost of slight performance loss than DM-OFDM. Although the detection complexity of the proposed GDM-OFDM is increased for the spectral efficiency gain, the computational complexity of the LLR detectors in the proposed GDM-OFDM, which is calculated to be $\mathcal{O}\left(l T\left(N_{A}+N_{B}\right)\right)$, is acceptable and may not be an critical issue for implementation. Moreover, under both channel conditions, the LLR detector presents marginal performance loss but reduces the computational complexity significantly compared with the ML detection, indicating that the LLR detector is more preferred in practical use.

The impact of higher-order constellations on the performance of the proposed GDM-OFDM is also investigated, as shown in Fig. 2, where two different 16-QAM constellations illustrated in [16] are applied. It can be seen that the proposed GDM-OFDM with $\mathcal{K}=\{1,3\}$ enhances the spectral efficiency by $0.22 \mathrm{bits} / \mathrm{s} / \mathrm{Hz}$ over DM-OFDM without performance loss, implying that the proposed GDM-OFDM outperforms conventional DM-OFDM using high-order constellations.

Additionally, the performances of GDM-OFDM and DMOFDM with and without the interleaving technique is presented in Fig. 3, where the frequency-selective Rayleigh fading channel is considered. At the BER level of $10^{-3}$, IGDMOFDM harvests on about $1.5 \mathrm{~dB}$ and $0.8 \mathrm{~dB}$ performance gains over GDM-OFDM and the interleaved DM-OFDM (IDMOFDM), respectively, indicating that the interleaving technique can improve the BER performance of index-modulated OFDM systems, and IGDM-OFDM is capable of enhancing the performance of IDM-OFDM with even higher spectral efficiency.

The performances of the coded GDM-OFDM, DM-OFDM and OFDM systems are shown in Fig. 4, where LDPC codes in IEEE 802.11 are utilized with the code length of 648 . BPSK is applied in OFDM, whilst two distinguishable BPSK schemes $\{1,-1\}$ and $\{\mathbf{j},-\mathbf{j}\}$ are used for index modulation. Two different code rates $1 / 2$ and $2 / 3$ are used in GDMOFDM, whilst the code rates for DM-OFDM and OFDM equal $2 / 3$ and $5 / 6$, respectively. It can be seen from Fig. 4 that GDM-OFDM still outperforms DM-OFDM and conventional OFDM in coded systems with improved spectral efficiency.

\section{CONCLUSION}

In this letter, GDM-OFDM is proposed, where subcarriers are partitioned into subblocks, and all the subcarriers in each OFDM subblock are modulated by two different constellation 


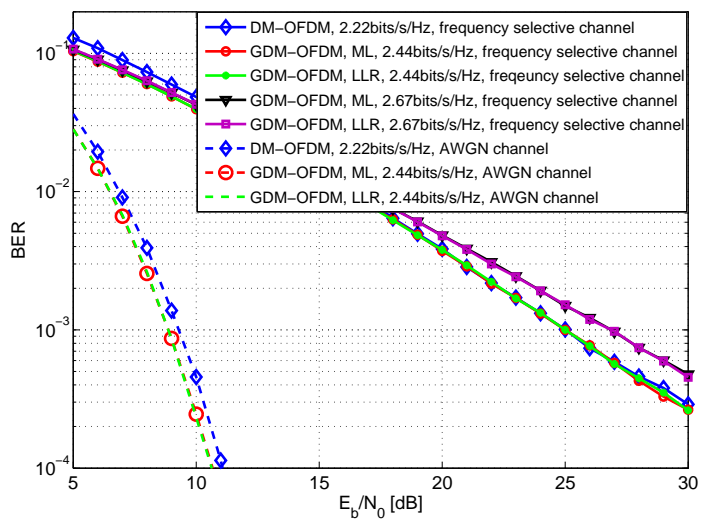

Fig. 1 Performance comparison between GDM-OFDM and DMOFDM using ML and LLR detectors under both the AWGN channel and the frequency-selective Rayleigh fading channel.

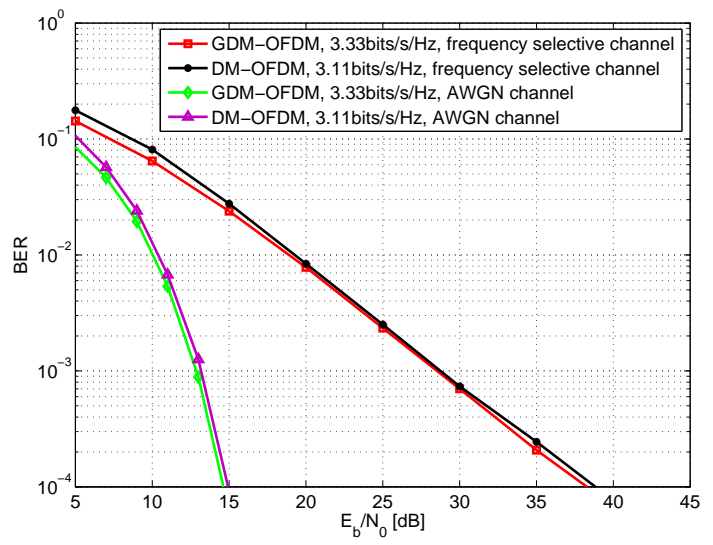

Fig. 2 Performance comparison between GDM-OFDM and DMOFDM using two 16-QAM constellations both the AWGN channel and the frequency-selective Rayleigh fading channel.

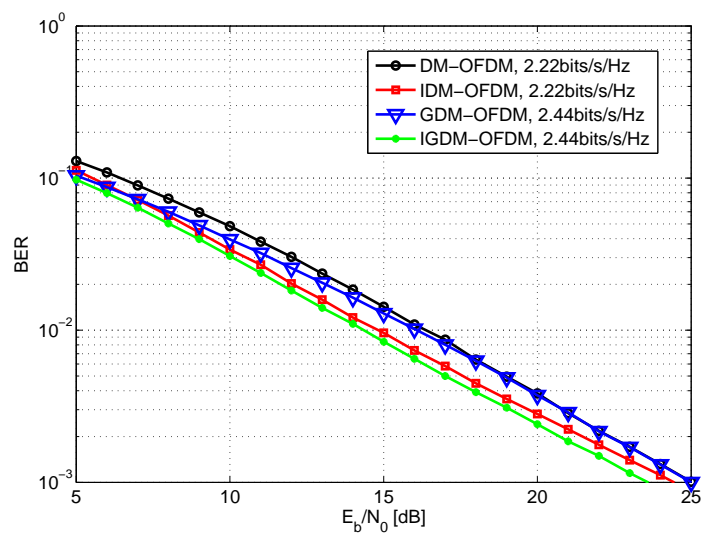

Fig. 3 Performances of GDM-OFDM and DM-OFDM with and without the interleaving technique under Rayleigh fading channel.

alphabets, conveying additional information bits through subcarrier indices. Unlike the classical DM-OFDM, the number of subcarriers modulated by either constellation alphabet is no longer fixed, yielding more possible realizations of OFDM subblocks. Hence GDM-OFDM is capable of significantly enhancing the spectral efficiency. Moreover, an interleaving

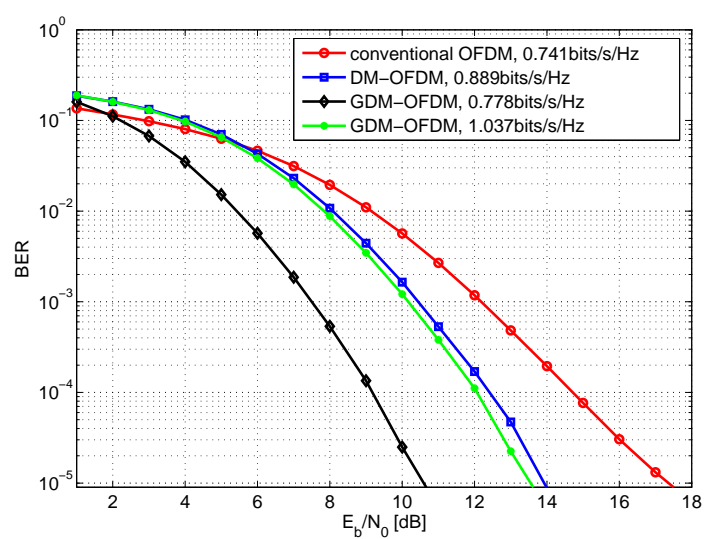

Fig. 4 Performance comparison between the LDPC-coded GDMOFDM, DM-OFDM, and conventional OFDM systems under the frequency-selective Rayleigh fading channel.

technique is employed to eliminate the correlation of FD channel coefficients under the frequency-selective Rayleigh fading channel, harvesting on additional diversity gain. The simulation results demonstrate that GDM-OFDM attains higher spectral efficiency than DM-OFDM at the cost of marginal performance loss, and IGDM-OFDM outperforms DM-OFDM in both the BER performance and the spectral efficiency.

\section{REFERENCES}

[1] G. Zhang, M. De. Leenheer, A. Morea, and B. Mukherjee, "A survey on OFDM-based elastic core optical networking," IEEE Commun. Surveys \& Tutorials, vol. 15, no. 1, pp. 65-87, Feb. 2013.

[2] L. Hanzo, M. Münster, B.J. Choi and T. Keller: OFDM and MC-CDMA for Broadband Multi-user Communications, WLANs and Broadcasting, John Wiley - IEEE Press, Jul. 2003.

[3] IEEE Std. 802.11-2012, Part 11: Wireless LAN Medium Access Control (MAC) and Physical Layer (PHY) Specifications (2012).

[4] 3GPP2 C.S0002-F v1.0, Physical Layer Standard for cdma2000 Spread Spectrum Systems (2012).

[5] T. Datta, H. S. Eshwaraiah, and A. Chockalingam, "Generalized space and frequency index modulation," IEEE Trans. Veh. Technol., vol. 65, no. 7, pp. 4911-4924, Jul. 2016.

[6] E. Basar, U. Aygolu, E. Panayirci, and H. V. Poor, "Orthogonal frequency division multiplexing with index modulation," IEEE Trans. Signal Processing, vol. 61, no. 22, pp. 5536-5549, Nov. 2013.

[7] E. Basar, "Index modulation techniques for $5 \mathrm{G}$ wireless networks," IEEE Commun. Mag., vol. 54, no. 7, pp. 168-175, Jul. 2016.

[8] N. Ishikawa, S. Sugiura, and L. Hanzo, "Subcarrier-index modulation aided OFDM - will it work?” IEEE Access, vol. 4, pp. 2580-2593, Jun. 2016.

[9] E. Basar, "Multiple-input multiple-output OFDM with index modulation," IEEE Signal Processing Lett., vol. 22, no. 12, pp. 2259-2263, Dec. 2015.

[10] E. Basar, "On multiple-input multiple-output OFDM with index modulation for next generation wireless networks," IEEE Trans. Signal Processing, vol. 64, no. 15, pp. 3868-3878, Aug. 2016.

[11] E. Basar and E. Panayirci, "Optical OFDM with index modulation for visible light communications," Proc. IEEE Int. Workshops on Opt. Wirel. Commun. (Istanbul, Turkey), Sept. 7-8, 2015, pp. 11-15.

[12] M. Wen, X. Cheng, L. Yang, Y. Li, X. Cheng, and F. Ji, "Index modulated OFDM for underwater acoustic communications," IEEE Commun. Mag., vol. 54, no. 5, pp. 132-137, May 2016.

[13] E. Basar, "OFDM with index modulation using coordinate interleaving," IEEE Wireless Commun. Lett., vol. 4, no. 4, pp. 381-384, Aug. 2015.

[14] Y. Xiao, S. Wang, L. Dan, X. Lei, P. Yang, and W. Xiang, "OFDM with interleaved subcarrier-index modulation," IEEE Commun. Lett., vol. 18, no. 8, pp. 1447-1450, Aug. 2014.

[15] R. Fan, Y. J. Yu, and Y. L. Guan, "Generalization of orthogonal frequency division multiplexing with index modulation," IEEE Trans. Wireless Commun., vol. 14, no. 10, pp. 5350-5359, Oct. 2015.

[16] T. Mao, Z. Wang, Q. Wang, S. Chen, and L. Hanzo, "Dual-mode index modulation aided OFDM," IEEE Access, to appear. 